\title{
How to improve gait and balance function in elderly individuals - compliance with principles of training
}

\author{
Lars I. E. Oddsson • Patrick Boissy • Itshak Melzer
}

Received: 3 November 2006 / Accepted: 15 February 2007 / Published online: 27 February 2007

(C) EGREPA 2007

\begin{abstract}
Most balance training regimens for elderly individuals focus on self-controlled exercises, although automatic postural responses after a balance perturbation are not under direct volitional control. We critically review the literature on this topic, and notice that several studies fail to comply with basic principles of training and therefore show little improvement in function. Some present the view that physical function in the too frail and too fit cannot be improved, which we instead argue would be the effect of nonspecific training programs. We propose a concept for balance training that incorporates voluntary exercises as well as perturbation and dual-task exercises to improve balance control. The program is performed on five different levels where levels 1-4 exercises focus on the skill to maintain balance and level 5 adds perturbation exercises that focus on the skill to recover balance as well as dual task exercises providing a cognitive load during execution
\end{abstract}

L. I. E. Oddsson $(\bowtie) \cdot$ I. Melzer

NeuroMuscular Research Center, Boston University,

19 Deerfield Street,

Boston, MA 02215, USA

e-mail: loddsson@bu.edu

P. Boissy

Research Center on Aging,

Sherbrooke Geriatric University Institute, Sherbrooke University,

Sherbrooke, Canada

I. Melzer

Sargent College of Health and Rehabilitation Sciences,

Boston University,

Boston, MA, USA

I. Melzer

Department of Physical Therapy, Faculty of Health Sciences,

Ben-Gurion University of the Negev,

Beer-Sheva, Israel of a balance motor task. Functional requirements for muscle strength and power are directly incorporated into the program. The feasibility of the concept has been demonstrated on elderly fallers. A randomized control trial is underway to investigate the effects on healthy elderly individuals. Further intervention studies using this concept are encouraged.

Keywords Exercise movement techniques $\cdot$ Posture . Rehabilitation

\section{Background}

Balance control is a foundation of our ability to move and function independently. A deterioration of balance function, as a consequence of disease or simply increasing age, will increase the occurrence of clinical balance problems as well as the risk of balance loss and falls. Falls are the leading cause of accidental death in the elderly population with more than 11,000 deaths as a result of falls each year [73] and hip fractures being the leading fall-related injury that causes prolonged hospitalization [69]. Hip fracture survivors experience a 10 to $15 \%$ decrease in life expectancy and a significant decline in overall quality of life [40]. Early ambulation after hip fracture, however, has been shown to be directly predictive of extended survival [36] indicating the importance of effective rehabilitation techniques that target balance function and early mobility. Unfortunately, a common view in the literature, as stated in a recent review study [78], is that physical function in individuals who are either "too frail" or "too fit" cannot be improved with training implying that rehabilitation for some patients as well as preventive training of healthy individuals would be futile. A contrary interpretation would be that a number of 
studies, aiming to improve function, have not complied with basic principles of physical training and exercise by assuming that strength training per se improves balance function. This is a view that is supported in a Cochrane Review by Latham et al. [41] of 62 randomized control trials including a total of 3,674 subjects showing no statistically significant effects of progressive strength training in elderly subjects on objective clinical measures of balance function (Berg Balance Score, Timed Up and Go, and Timed position holding), or on physical disability measures [41]. Additional support for the view that strength training does not improve balance function on its own is provided in a recent Cochrane Review by Gillespie et al. [25] showing that successful interventions designed to reduce the incidence of falls in elderly individuals have been multidisciplinary/multifactorial and have incorporated muscle strengthening and balance training. Tai Chi as a balance intervention has been studied quite extensively ever since the now classical work by Wolf et al. suggested that Tai Chi training decreased the fear of falling, although it had little effect on computerized sway measures [82]. More recent studies have confirmed beneficial effects of Tai Chi participation on balance function including reduction in fear of falling [74] as well as physical function and actual falls $[47,83]$. However, results of Tai Chi training is commonly compared to untrained controls, simple stretching routines, wellness education programs, or nonspecific and nonfunctional balance interventions making it difficult to really assess whether Tai Chi is any better than a well-designed balance specific and functionally focused training regimen [46]. Furthermore, there is often a "spiritual magical aura" surrounding Tai Chi that subjects may or may not appreciate. Consequently, as pointed out by $\mathrm{Li}$ et al. [46], "This calls for controlled and systematic evaluation of the characteristics of movement kinematics, kinetics, and neuromuscular activity in Tai Chi and the need for better understanding of the integration of muscular, skeletal, and neural functioning and their interplay in the execution of Tai Chi movements." Such studies should put Tai Chi in the context of known principles of training reflecting physiological as well as psychological concepts that therapists can apply in the design of individually customized exercise regimens.

Although the benefits of exercise with respect to general health, strength, and fitness as well as quality of life have been documented in the literature for decades, physical exercise as a technique in rehabilitation or as a measure to prevent injury is not always implemented correctly. Some published studies targeting balance training, particularly in the elderly population, have not fully complied with basic principles of training used in exercise prescription. Principles of physical training and exercise prescription include, e.g., awareness, continuity, motivation, overload, periodicity, progression, and specificity. These important concepts are well established and accepted in the exercise physiology literature (e.g., $[1,2,27,72,79])$, and any successful athlete must abide and live by these rules to maintain and/or improve function and performance. However, these fundamental principles of successful exercise prescription apply to anyone regardless of age, sex, or level of fitness [68], even patients. Thus, an exercise intervention targeting a certain function must provide a challenge/overload to the system and be progressive as well as specific to this function. Otherwise, a training effect should not be expected. As recognized by Welsch et al. [81], the basic principles of prescribing exercise are also appropriate for persons with disease. The difference is the manner in which these principles are applied [81].

Although some studies have investigated the effect of exercise on balance impairment in the elderly, the training principles behind these programs are sometimes unclear. For example, a recent study of fall prevention performed training only in sitting assuming effects would transfer to standing. Because of the lack of specificity in this model, it is not very surprising that no effects of the intervention were found [55]. Other studies have had similar problems (e.g., [31, 32]), and few have realized the importance of perturbation exercises for improving balance function. $\mathrm{Hu}$ and Woollacott [33, 34], however, used a multisensory training model specific to balance function and the interaction between visual, vestibular, and somatosensory systems and found specific improvements in balance function after a short training period (cf. [29, 35, 84]). More recently, there have been well-designed studies that include dual-task conditions as well as perturbations [71, $76,77]$ or that utilize a custom designed program for each patient [3], which by definition would improve specificity of the intervention as well as the effect of the intervention.

A common misconception appears to be that strength or even cardiovascular training per se should improve balance function (e.g., $[10,11,31,32,62,75,80])$. There is undoubtedly a documented relationship between falls and muscle strength in the elderly (e.g., [9]). However, results are controversial, and other studies show minimal or even no differences in strength between fallers and nonfallers [15]. Although several studies have demonstrated improvements in balance function after strength training, it is usually related to tasks where muscle strength is a dominant and obvious component of the task (e.g., sit to stand, [38, 70], rapid backward walking [62]). In fact, balance improvement related to strength training appears to occur primarily in subjects with severely compromised strength and muscle function (e.g., [11, 20, 57]). In addition, several well-designed studies have not found an interaction between balance and strength (e.g., [35, 84, 85]). This illustrates the importance of an accurate definition of 
muscle strength and balance function. In the context of the current paper, strength is defined as the capacity to produce torque around a certain joint, whereas balance is defined as a task-specific multijoint skill that relies on the interaction of several physiological sensory systems, including the neuromuscular, visual, vestibular, and somatosensory ones. To be functionally useful, strength should preferably be designed into the balance training intervention, an approach taken in the current project. In addition, to comply with basic principles of training, the design of specific and functional balance training programs for any category of subjects requires an understanding of how voluntary and postural movements interact as well as how the postural control system reacts to balance perturbations.

\section{Voluntary movements and postural control}

Because of the multilink structure of the human body, any voluntary movement by itself will impose a perturbation of posture. To compensate for this internal perturbation, our voluntary movements are preceded and accompanied by anticipatory postural adjustments. These involuntary "automatic" movements are smoothly incorporated into our movement repertoire to ensure accurate and harmonious motion [24, 66]. In fact, the activation of muscles controlling these postural adjustments precedes the activation of voluntary muscle activity as first demonstrated by Belenkii et al. [4]. The interaction between voluntary movements and anticipatory postural adjustments has since been studied extensively during different upper limb movements [6-8, 23, 44, 45, 50], leg movements [37, 58] as well as trunk movements [13, 14, 63-67]. The postural synergies triggered before the onset of voluntary movements are flexible and task specific, i.e., a certain voluntary movement may be associated with different automatic postural adjustments depending on the context of the task $[26,42,66,67]$. The execution of a voluntary step is preceded by an anticipatory postural adjustment that shifts body weight over to the upcoming stance leg [53]. To prevent a fall, a rapid step execution is a highly important skill that appears to deteriorate with increasing age [52], in particular when tested under conditions of cognitive stress [56]. An example of anticipatory postural adjustments is shown in the upper row of Fig. 1. Note how the woman on the left in preparation of receiving the ball rolling towards her leans the upper body towards the left away from the right leg she plans to lift to stop the ball. The man on the right illustrates the lack of the ability to perform this preparatory movement. Note how he tends to fall towards the right leg when he lifts it without first shifting his weight over to the opposite leg. These postural synergies are trained through voluntary exercises, and they are the traditional target of most well-designed balance training regimes.
Voluntary movements and external perturbations of posture

External perturbations of posture, such as a slip or a trip, trigger automatic postural responses with a delay of about $100 \mathrm{~ms}$, which act to restore equilibrium. The responses are specific to the size, type, and direction of the imposed perturbation [12, 16, 30, 39, 54, 59, 61]. Minor perturbations at the feet trigger what has been termed an "ankle-strategy," i.e., muscles around the ankle create a torque that helps regain equilibrium. Larger perturbations will trigger a "hipstrategy," which initiates specific hip movements that help maintain balance [30]. If the subject cannot regain balance, a step will be initiated indicating that the other strategies have failed to recover equilibrium, although when required, a step strategy can be initiated immediately (e.g., [19, 54]). These responses have been studied extensively in healthy human subjects [16-18, 59, 61, 86] as well as in elderly [33, 34, 43, $50,51]$ and in different patient populations [33, 34, 43, 50, 51]. Similar types of automatic responses occur after postural perturbations during locomotion [60], during sitting [22, 28] as well as in standing cats $[48,49]$. These recovery strategies are not under direct volitional control, and thus, they cannot be trained through voluntary exercises alone. In spite of this, most balance training programs only include voluntarily controlled exercises. Support for a contrarian view is found in recent reports on effects from Tai Chi training suggesting shorter reflex responses after long-term (1-3 years) training [21], although such an effect was only inferred from past training.

\section{Program design}

In an attempt to address some of the aforementioned concerns, a balance training program based on a framework of principles of exercise training and postural control has been designed. The program utilizes different types of balls, including large "Balance Balls," as tools to target sitting and standing balance control. The concept, however, can be implemented with other training tools by a skilled instructor. The program is performed on five different levels where each level reflects different demands on the postural control system. Levels 1-4 include exercises that are focused on the skill to maintain balance (voluntary control), whereas level 5 also includes perturbation exercises that focus on the skill to recover balance (automatic postural corrections). The program is described as a concept with examples to indicate the kind of exercise and the type of skills they intend to develop as well as the overall level of difficulty a certain level should represent. The program should be implemented by an instructor or therapist who, based on each individual subjects' background and current skill/ fitness level, can assess at what level a subject should begin 
Fig. 1 Upper row, the individual on the left performs a normal preparatory postural adjustment to lift her leg before receiving the ball. The individual on the right lacks this preparatory postural adjustment and loses balance when lifting the leg. See text for details. Middle row, examples of level 1 (left) and level 2 exercises (right). Lower row, examples of level 3 (left) and level 4 exercises (right)
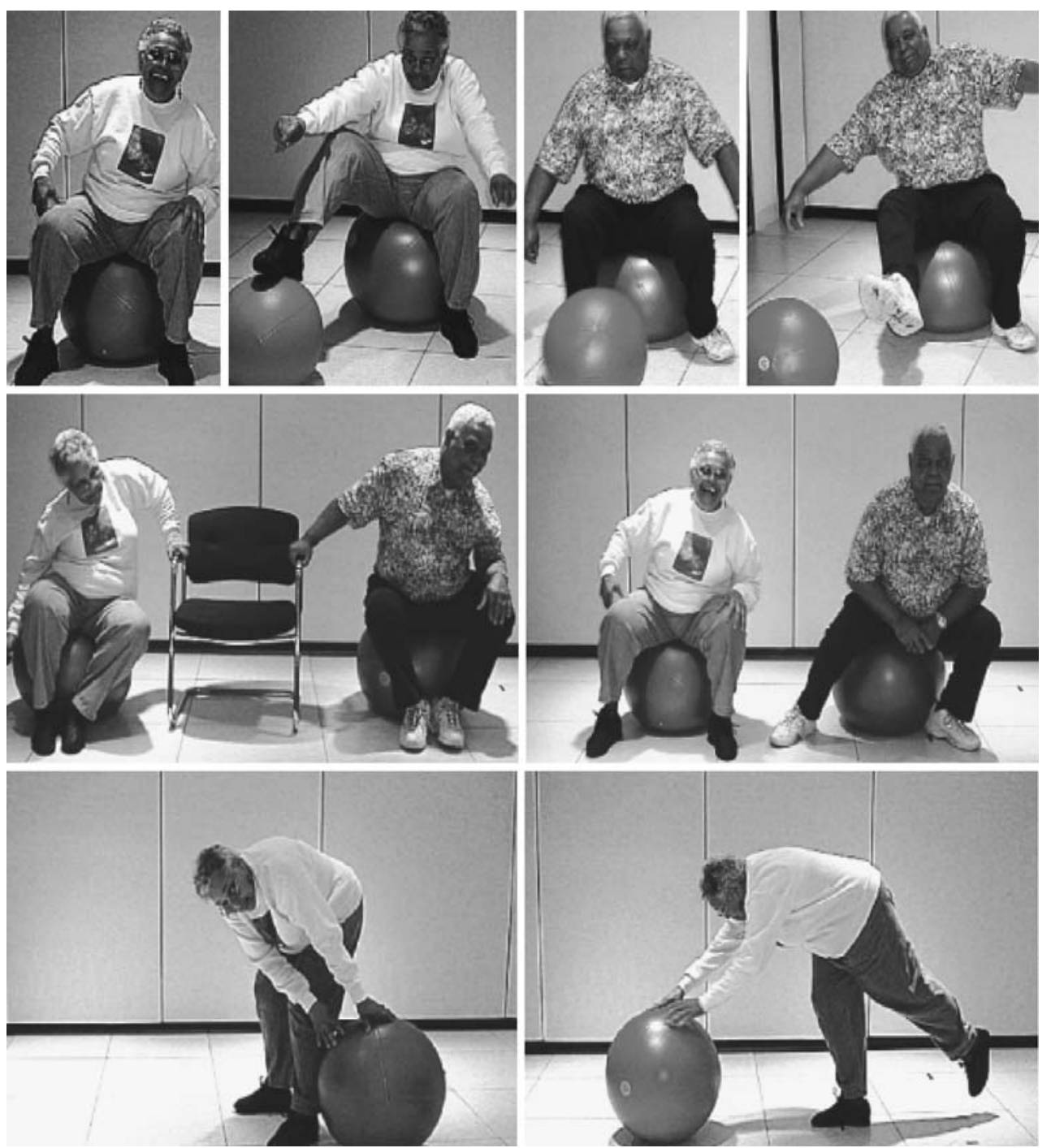

the training program to maximize compliance and outcome and maintain safety.

Any form of physical training, either learning and perfecting a sport skill or rehabilitating after an injury or disease, is a process that incorporates both physiological and psychological factors. These factors must harmonize on an individual level for the training process to be successful. A very important role of the instructor is to monitor the progression of the training process on an individual level and to adapt the training program to optimize outcome and maximize performance. When needed, the instructor should be able to modify the execution of an exercise "on-the-fly" to customize the difficulty on an individual level. Factors that can be used to modify the demand of the task include open and closed eyes, stance width during standing and sitting, range of motion, movement speed (challenging power production), single and double leg support, bouncing on the ball, unstable support surface, etc. This process requires the interaction with an experienced therapist, instructor, or coach and/or should not be based on a "cookbook" approach to training. As in any type of training, for improvement to occur, it is crucial to maintain a progressive and specific training load. In addition, variability is important to keep subjects motivated and excited to pursue the training.

The goal of each session in the current program is to constantly challenge the postural control system with exercises that incorporate elements related to the demands of normal activities of daily living. Such elements include single leg standing, weight transfer between feet, during feet in place, and change of support area as well as different reaching and bending movements of the trunk while standing and sitting. The concept of balance or body awareness should be incorporated throughout the exercise period. For example, subjects should be encouraged to feel how they are balanced when they are sitting and standing and also focus on smooth and comfortable movements while shifting their weight. Instructing them to focus on where they feel pressure under their feet, how it moves, and where body parts are located with respect to each other as 
well as the feet are important aspects of the training. Such visualization of balance may be most efficient when the subjects have no visual feedback, i.e., closed eyes. Thus, in addition to providing an increased level of balance challenge, visual occlusion allows the subject to focus on better feeling how they are balanced, thus enhancing the experience and interpretation of somatosensory and vestibular balance cues. Exercises can also be modified to include components more specific for vestibular and vestibulooccular function. For example, subjects can be instructed to move their head while they are sitting and bouncing on the ball. Head movements can be pitch, yaw, or roll. During head movements, subjects can be instructed to focus on specific objects and even read text out loud to further enhance attention to the task. In addition, it is also important to promote skill awareness among the subjects. This simply means that subjects should be encouraged to understand the goal of an exercise with respect to balance control and to relate this goal to their personal skill level. Eventually, some subjects may be able to "coach" themselves and continue training on their own.

The number of subjects that can be safely managed by one instructor depends on the skill level of the subjects as well as the difficulty of the exercises performed. Some exercises are by design challenging for balance, and in case a fall occurs, proper support and assistance from an instructor must be instantly available to eliminate risk of injury. It should be noted, however, that up to the point of balance loss, provided external support by the instructor should be avoided to allow the natural balance response to fully execute. Providing support "too early" may alter the response and decrease or alter the learning effect. It is important that the subject, when performing such challenging exercises, feels completely safe so that their confidence steadily builds during the training process. Examples of correctly provided support during challenging exercises are provided in Fig. 2. In a group setting for healthy elderly individuals, we have managed 10 12 individuals with one main instructor and one supporting instructor. Highly frail and/or unbalanced individuals will require one-on-one interaction for safe execution of challenging exercises. However, small groups of 2-4 individuals can be managed by one instructor once level $1-2$ exercises can be safely performed, allowing the instructor to move around the group and assist one subject at a time to perform more challenging exercises.

Each session should include an initial warm-up and stretch period and some strengthening/conditioning exercises as well as a cool down and relax period at the end where the instructor may reemphasize some of the important aspects of the session, especially with respect to body and skill awareness. The use of relaxing music or soothing sounds can be used to create a good ambient atmosphere during the session.
Examples of exercises at each level

\section{Level 1: sitting and standing exercises with external} support

Exercises on this level may represent little challenge to the postural control system. The goal of the training at this level is mainly directed towards a cognitive understanding of the exercises and an improvement of self-confidence for exercises on higher levels. These exercises can also be used as safe "rest" after more difficult bouts of exercise. Some general effects on strength, coordination, and conditioning are expected. Everyone should progress through this level as soon as possible. However, elements from exercises at this level will be included also on the other levels. An example of a level 1 exercise is shown in Fig. 1, left middle row. Additional examples are provided below.

1. Sit on ball, wide stance, with support with one hand from the wall or a fixed object. Repeat with narrow stance and support.

2. Sit on ball, wide stance, with support, and shift weight left and right as far as possible. Repeat with narrow stance.

3. Sit on ball and rotate trunk left and right as far as possible, with support.

4. Sit on ball, wide stance, with support, and lift one foot at a time. Repeat in narrow stance.

\section{Level 2: sitting exercises without external support}

The training on this level mainly addresses balance of the upper body during sitting, and it will challenge postural control in a predictable and controlled manner through voluntary movements. Exercises are similar to level 1 but without external support (cf. Fig. 1, middle row right). For the purpose of activating relevant associated postural adjustments, which are the target of the training at this level, it is more beneficial to execute an exercise slowly with small amplitude rather than using external support because this will completely change the set of muscles that are recruited for balance control during the task. Thus, to progress to this level, the participants must perform exercises with no external support. Depending on the skill of the subject, these exercises can also be used as safe "rest" after more challenging bouts of exercise.

Level 3: standing exercises including double leg stance and no external support

Exercises on this level incorporate specific components that reflect balance requirements of common activities of daily 

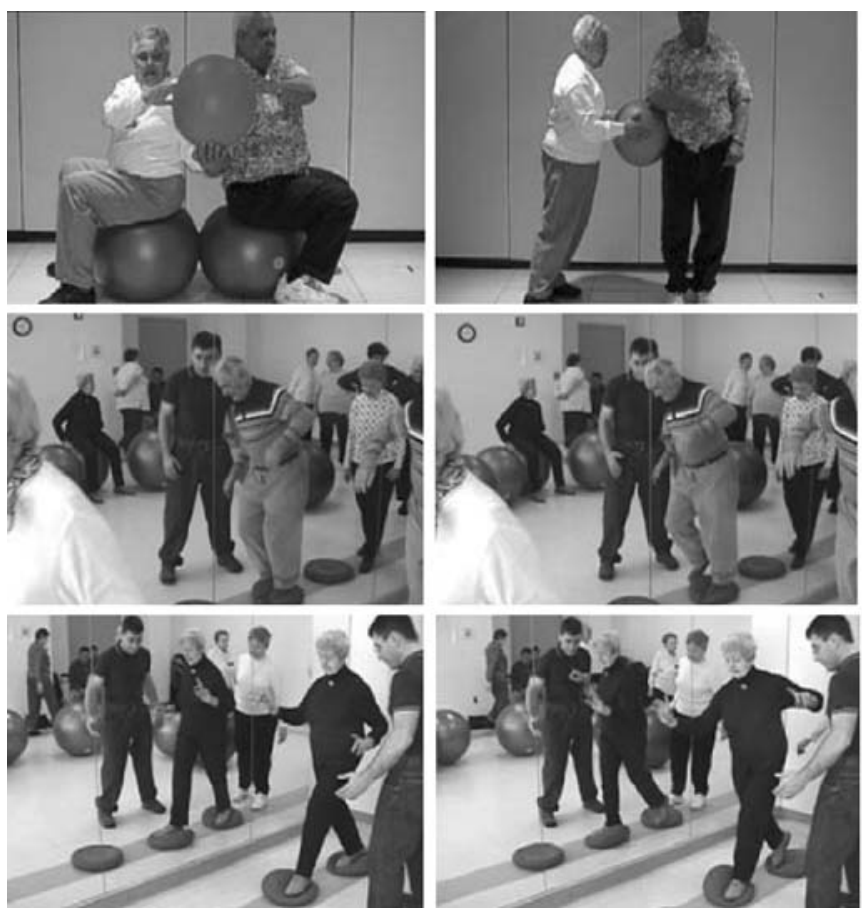

Fig. 2 Upper row, examples of partner exercises that incorporate elements from level 5 where the subjects must negotiate expected and unexpected external perturbations of posture. Note that the subject is out of balance and in the process of executing a step in the third
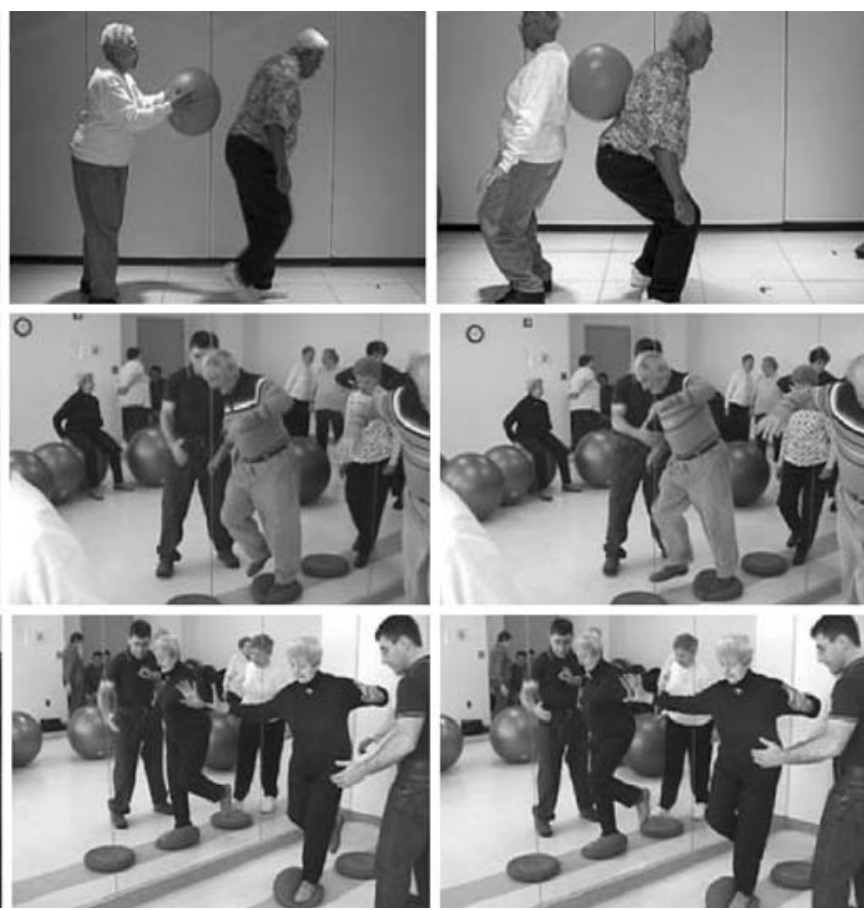

picture. Middle and lower rows, individual level 5 exercise involving walking on an unstable surface. Note how the two individuals lose and recover balance. Furthermore, note the instructor's attention and readiness level, and that external support is only provided if necessary living. This includes weight shifts laterally between the two feet as well as forward and backward (Fig. 1, lower row left). Assistance and support can be used in case anyone feels uncomfortable in the initial phase of the training, although when support is provided, the exercise would be at level 1. Similar to exercises at level 2, participants should be encouraged to perform exercises with no or only temporary external support. Subjects should be brought to this level of training as soon as their ability allows them to. Examples of exercises include:

1. Wide parallel stance, roll ball in front of body from left to right with no weight shift between legs.

2. Wide parallel stance, roll ball in a circle around the body with no weight shift between legs. Repeat with weight shift. Repeat in narrow stance.

\section{Level 4: standing exercises including single leg stance,} gait, and no external support

Exercises on this level are similar to level 3; however, they incorporate single leg support, an important component of functional independent ambulation (Fig. 1, lower row right). This level will also include walking exercises with turns and rotations as well as lateral and forward/backward walking. Assistance and support may be used in case anyone feels uncomfortable in the initial phase of the training. Examples of exercises include:

1. Step stance, roll ball straight forward and then back, lift rear leg when forward, and vice versa.

2. Multidirectional lunges: Step with right foot forward, yield slowly, and push back distinctly up to parallel stance. Repeat for right $45,90,135$, and $180^{\circ}$. Keep ball on left side for support if needed. Repeat with left foot and ball on right side. To further challenge power production, a more demanding version of the same task can be performed with a faster rebound and no yield.

\section{Level 5: perturbation exercises, reactive and proactive} responses

Exercises on this level include different forms of external perturbations, expected as well as unexpected ones that require a proactive or reactive response by the subject. Perturbations requiring a proactive response by the subject may be applied during different forms of partner or group exercises through the exchange of objects such as different size and weight balls that may be rolling on the floor or handed over by another participant (Fig. 2, top row left). Exercises incorporating both reactive and proactive responses include, e.g., two participants standing back to 
back supporting a ball between them (Fig. 2, top row right). In addition, the instructor or a partner could also apply unexpected external perturbations that require a reactive response. Participants may also be exposed to exercises where they will be encouraged to resist a balance perturbation and avoid stepping, or to execute one or several steps as quickly as possible (Fig. 2, top row middle pictures). A rapid step execution is a very important protective reaction when balance is lost [56]. It is important to note that these exercises should be customized to each subject's ability. They should be challenging but never dangerous thus building self-confidence and skill. However, note that subjects should temporarily lose their balance when exercising at this level (cf. Fig. 2, middle and lower rows). This is required to trigger the postural responses that are the target of the training. An additional form of exercise on this level incorporates cognitive stress and specific requirements for attention while performing a motor task, commonly walking. For example, subjects may be walking and reading a text out loud (one at a time or everyone at the same time). At the same time, they must pay attention to commands provided randomly by the instructor to, for example, stop, take a step in a certain direction, etc. They may also receive a light push by the instructor simulating an external balance perturbation that they must react to.

Examples of level 5 exercises include:

1. Exercises from level 2 with perturbations provided by the instructor or a partner.

2. Sit and bounce on ball, get up to standing after bounce, and get up and walk after bounce.

3. Resist light push at trunk or hip level and keep feet in place (center of mass perturbation). Repeat with step execution, one step or several small ones. Emphasize rapid response (Fig. 2, top row middle pictures).

4. Walk across unstable surface (base of support perturbation; Fig. 2, lower two rows).

\section{Conclusion}

The feasibility of this training concept has been tested in a pilot study on a group of elderly fallers [5]. Early results indicate improvements in functional status as well as postural control variables [5]. Compliance to the exercise program is excellent and participants have reported that they feel safe while performing the program and that the exercises are challenging. All subjects have stated that they would recommend the program to friends and family. Participants also reported that the training sessions provided a good group dynamic atmosphere favorable to socialization. A randomized controlled trial has been conducted to investigate effects of the proposed training program on gait and balance function in healthy elderly individuals. We believe the proposed training technique merits additional study in future clinical intervention trials.

Acknowledgments This work was supported by the Retirement Research Foundation, the Veterans Administration (E-2184-R) and NIH (5R21HD050655-02).

\section{References}

1. No authors listed (1998) American College of Sports Medicine Position Stand. Exercise and physical activity for older adults. Med Sci Sports Exerc 30(6):992-1008

2. No authors listed (1998) American College of Sports Medicine Position Stand. The recommended quantity and quality of exercise for developing and maintaining cardiorespiratory and muscular fitness, and flexibility in healthy adults. Med Sci Sports Exerc 30 (6):975-991

3. Badke MB et al (2004) Outcomes after rehabilitation for adults with balance dysfunction. Arch Phys Med Rehabil 85(2):227-233

4. Belen'kii VE, Gurfinkel VS, Pal'tsev EI (1967) Control elements of voluntary movements. Biofizika 12(1):135-141

5. Boissy P, Yurkow J, Chopra A, Oddsson L, De Luca C (2000) Balance training in the elderly using Swiss ball: a pilot study. Arch Phys Med Rehabil 81(10):1463

6. Bouisset S, Zattara M (1990) Segmental movement as a perturbation to balance? Facts and concepts. In: Winters J, Woo S (eds) Multiple muscle systems: biomechanics and movement organization. Springer, Berlin, pp 498-506

7. Bouisset S, Zattara M (1981) A sequence of postural movements precedes voluntary movement. Neurosci Lett 22:263-270

8. Brown JE, Frank JS (1987) Influence of event anticipation on postural actions accompanying voluntary movement. Exp Brain Res 67(3):645-650

9. Buchner DM (1997) Preserving mobility in older adults. West J Med 167(4):258-264

10. Buchner DM et al (1993) The Seattle FICSIT/MoveIt study: the effect of exercise on gait and balance in older adults. J Am Geriatr Soc 41(3):321-325

11. Chandler JM et al (1998) Is lower extremity strength gain associated with improvement in physical performance and disability in frail, community-dwelling elders? Arch Phys Med Rehabil 79(1):24-30

12. Cordo PJ, Nashner LM (1982) Properties of postural adjustments associated with rapid arm movements. J Neurophysiol 47(2):287-302

13. Crenna P, Frigo C (1991) A motor programme for the initiation of forward-oriented movements in humans. J Physiol 437:635-653

14. Crenna $P$ et al (1987) Forward and backward axial synergies in man. Exp Brain Res 65(3):538-548

15. Daubney ME, Culham EG (1999) Lower-extremity muscle force and balance performance in adults aged 65 years and older. Phys Ther 79(12): 1177-1185

16. Diener HC et al (1983) Variability of postural "reflexes" in humans. Exp Brain Res 52(3):423-428

17. Dietz V et al (1992) Regulation of bipedal stance: dependency on "load" receptors. Exp Brain Res 89(1):229-231

18. Dietz V et al (1989) Human postural reflexes and gravity-an under water simulation. Neurosci Lett 106(3):350-355

19. Do MC, Breniere Y, Brenguier P (1982) A biomechanical study of balance recovery during the fall forward. J Biomech 15(12):933-939

20. Fiatarone MA et al (1993) The Boston FICSIT study: the effects of resistance training and nutritional supplementation on physical frailty in the oldest old. J Am Geriatr Soc 41(3):333-337 
21. Fong SM, Ng GY (2006) The effects on sensorimotor performance and balance with Tai Chi training. Arch Phys Med Rehabil 87(1):82-87

22. Forssberg H, Hirschfeld H (1994) Postural adjustments in sitting humans following external perturbations: muscle activity and kinematics. Exp Brain Res 97(3):515-527

23. Friedli WG, Hallett M, Simon SR (1984) Postural adjustments associated with rapid voluntary arm movements 1 . Electromyographic data. J Neurol Neurosurg Psychiatry 47(6):611-622

24. Gahery Y, Massion J (1981) Co-ordination between posture and movement. Trends Neurosci 4:199-203

25. Gillespie L et al (2004) Interventions for preventing falls in elderly people (Cochrane review). In: The Cochrane Library, issue 1. Wiley, Chichester, UK

26. Gurfinkel V, Latash M (1979) Segmental postural mechanisms and reversal of muscle reflexes. Agressiologie 20:145-146

27. Harre D (1982) Principles of sports training, introduction to the theory and methods of training. Sportverlag, Berlin, Germany

28. Hirschfeld H, Forssberg H (1994) Epigenetic development of postural responses for sitting during infancy. Exp Brain Res 97 (3):528-540

29. Hocherman S, Dickstein R, Pillar T (1984) Platform training and postural stability in hemiplegia. Arch Phys Med Rehabil 65 (10):588-592

30. Horak FB, Nashner LM (1986) Central programming of postural movements: adaptation to altered support-surface configurations. J Neurophysiol 55(6):1369-1381

31. Hornbrook MC, Stevens VJ, Wingfield DJ (1993) Seniors' program for injury control and education. J Am Geriatr Soc 41 (3):309-314

32. Hornbrook MC et al (1994) Preventing falls among communitydwelling older persons: results from a randomized trial. Gerontologist 34(1):16-23

33. $\mathrm{Hu}$ MH, Woollacott MH (1994) Multisensory training of standing balance in older adults: I. Postural stability and one-leg stance balance. J Gerontol 49(2):M52-M61

34. Hu MH, Woollacott MH (1994) Multisensory training of standing balance in older adults: II. Kinematic and electromyographic postural responses. J Gerontol 49(2):M62-M71

35. Judge JO, Whipple RH, Wolfson LI (1994) Effects of resistive and balance exercises on isokinetic strength in older persons. J Am Geriatr Soc 42(9):937-946

36. Kamel HK et al (2003) Time to ambulation after hip fracture surgery: relation to hospitalization outcomes. J Gerontol Ser A Biol Sci Med Sci 58(11):1042-1045

37. Kasai T, Kawai K (1994) Quantitative EMG analysis of anticipatory postural adjustments of voluntary contraction of leg muscles in standing man. Electroencephalogr Clin Neurophysiol 93(3):184-187

38. Kaya BK, Krebs DE, Riley PO (1998) Dynamic stability in elders: momentum control in locomotor ADL. J Gerontol Ser A Biol Sci Med Sci 53(2):M126-M134

39. Keshner EA, Woollacott MH, Debu B (1988) Neck, trunk and limb muscle responses during postural perturbations in humans. Exp Brain Res 71(3):455-466

40. King MB, Tinetti ME (1996) A multifactorial approach to reducing injurious falls. Clin Geriatr Med 12(4):745-759

41. Latham $\mathrm{N}$ et al (2004) Progressive resistance strength training for physical disability in older people (Cochrane Review). In: The Cochrane Library, issue 3. Wiley, Chichester, UK

42. Layne CS, Abraham LD (1991) Interactions between automatic postural adjustments and anticipatory postural patterns accompanying voluntary movement. Int J Neurosci 61(3-4):241-254

43. Ledin $T$ et al (1990) Effects of balance training in elderly evaluated by clinical tests and dynamic posturography. J Vestib Res 1(2):129-138
44. Lee W (1980) Anticipatory control of postural and task muscles during rapid arm flexion. J Mot Behav 12:185-196

45. Lee WA, Buchanan TS, Rogers MW (1987) Effects of arm acceleration and behavioral conditions on the organization of postural adjustments during arm flexion. Exp Brain Res 66 (2): $257-270$

46. Li $\mathrm{F}$ et al (2005) Tai Chi and fall reductions in older adults: a randomized controlled trial. J Gerontol Ser A Biol Sci Med Sci 60 (2):187-194

47. Lin MR et al (2006) Community-based tai chi and its effect on injurious falls, balance, gait, and fear of falling in older people. Phys Ther 86(9):1189-1201

48. Macpherson JM (1988) Strategies that simplify the control of quadrupedal stance. I. Forces at the ground. J Neurophysiol 60 (1):204-217

49. Macpherson JM (1988) Strategies that simplify the control of quadrupedal stance. II. Electromyographic activity. J Neurophysiol 60(1):218-231

50. Maki BE (1993) Biomechanical approach to quantifying anticipatory postural adjustments in the elderly. Med Biol Eng Comput 31(4):355-362

51. Maki BE, Holliday PJ, Topper AK (1994) A prospective study of postural balance and risk of falling in an ambulatory and independent elderly population. J Gerontol 49(2):M72-M84

52. Maki BE, McIlroy WE (2006) Control of rapid limb movements for balance recovery: age-related changes and implications for fall prevention. Age Ageing 35(Suppl 2):ii12-ii18

53. Maki BE, Mcllroy WE (1997) The role of limb movements in maintaining upright stance: the "change-in-support" strategy. Phys Ther 77(5):488-507

54. McIlroy WE, Maki BE (1993) Changes in early 'automatic' postural responses associated with the prior-planning and execution of a compensatory step. Brain Res 631(2):203-211

55. McMurdo ME, Millar AM, Daly F (2000) A randomized controlled trial of fall prevention strategies in old peoples' homes. Gerontology 46(2):83-87

56. Melzer I, Oddsson LI (2004) The effect of a cognitive task on voluntary step execution in healthy elderly and young individuals. J Am Geriatr Soc 52(8):1255-1262

57. Moxley Scarborough D, Krebs DE, Harris BA (1999) Quadriceps muscle strength and dynamic stability in elderly persons. Gait Posture 10(1):10-20

58. Nardone A, Schieppati M (1988) Postural adjustments associated with voluntary contraction of leg muscles in standing man. Exp Brain Res 69(3):469-480

59. Nashner LM (1976) Adapting reflexes controlling the human posture. Exp Brain Res 26(1):59-72

60. Nashner LM (1980) Balance adjustments of humans perturbed while walking. J Neurophysiol 44(4):650-664

61. Nashner LM (1977) Fixed patterns of rapid postural responses among leg muscles during stance. Exp Brain Res 30(1):13-24

62. Nelson ME et al (1994) Effects of high-intensity strength training on multiple risk factors for osteoporotic fractures. A randomized controlled trial. JAMA 272(24):1909-1914

63. Oddsson L (1988) Co-ordination of a simple voluntary multi-joint movement with postural demands: trunk extension in standing man. Acta Physiol Scand 134(1):109-118

64. Oddsson L, Thorstensson A (1987) Fast voluntary trunk flexion movements in standing: motor patterns. Acta Physiol Scand 129 (1):93-106

65. Oddsson L, Thorstensson A (1986) Fast voluntary trunk flexion movements in standing: primary movements and associated postural adjustments. Acta Physiol Scand 128(3):341-349

66. Oddsson LI (1990) Control of voluntary trunk movements in man. Mechanisms for postural equilibrium during standing. Acta Physiol Scand Suppl 595:1-60 
67. Pedotti A et al (1989) Postural synergies in axial movements: short and long-term adaptation. Exp Brain Res 74(1):3-10

68. Petrie D, Matthews LS, Howard WH (1996) Prescribing exercise for your patient. Md Med J 45(8):632-637

69. Rabin DW (1995) Falls and gait disorders. In: Abrams WB, Beers $\mathrm{MH}$, Berkow R (eds) The Merck manual of geriatrics. Merck Sharp \& Dohme Research Laboratories, Rahway, NJ, pp 65-78

70. Riley PO, Krebs DE, Popat RA (1997) Biomechanical analysis of failed sit-to-stand. IEEE Trans Rehabil Eng 5(4):353-359

71. Rogers MW et al (2003) Step training improves the speed of voluntary step initiation in aging. J Gerontol Ser A Biol Sci Med Sci 58(1):46-51

72. Sale D, MacDougall D (1981) Specificity in strength training: a review for the coach and athlete. Can J Appl Sport Sci 6(2):87-92

73. Sattin RW (1992) Falls among older persons: a public health perspective. Annu Rev Public Health 13:489-508

74. Sattin RW et al (2005) Reduction in fear of falling through intense tai chi exercise training in older, transitionally frail adults. J Am Geriatr Soc 53(7):1168-1178

75. Sauvage LR Jr et al (1992) A clinical trial of strengthening and aerobic exercise to improve gait and balance in elderly male nursing home residents. Am J Phys Med Rehabil 71(6):333-342

76. Shimada $\mathrm{H}$ et al (2004) New intervention program for preventing falls among frail elderly people: the effects of perturbed walking exercise using a bilateral separated treadmill. Am J Phys Med Rehabil 83(7):493-499

77. Silsupadol $P$ et al (2006) Training of balance under single- and dual-task conditions in older adults with balance impairment. Phys Ther 86(2):269-281
78. Skelton DA, Beyer N (2003) Exercise and injury prevention in older people. Scand J Med Sci Sports 13(1):77-85

79. Tanaka H, Swensen T (1998) Impact of resistance training on endurance performance. A new form of cross-training? Sports Med 25(3):191-200

80. Topp R et al (1993) The effect of a 12-week dynamic resistance strength training program on gait velocity and balance of older adults. Gerontologist 33(4):501-506

81. Welsch MA et al (1994) Using the exercise test to develop the exercise prescription in health and disease. Prim Care 21(3):589609

82. Wolf SL et al (1997) The effect of Tai Chi Quan and computerized balance training on postural stability in older subjects. Atlanta FICSIT Group. Frailty and Injuries: Cooperative Studies on Intervention Techniques. Phys Ther 77(4):371-381 (discussion 382-384)

83. Wolf SL et al (2006) The influence of intense Tai Chi training on physical performance and hemodynamic outcomes in transitionally frail, older adults. J Gerontol Ser A Biol Sci Med Sci 61 (2):184-189

84. Wolfson L et al (1996) Balance and strength training in older adults: intervention gains and Tai Chi maintenance. J Am Geriatr Soc 44(5):498-506

85. Wolfson L et al (1993) Training balance and strength in the elderly to improve function. J Am Geriatr Soc 41(3):341-343

86. Woollacott MH, von Hosten C, Rosblad B (1988) Relation between muscle response onset and body segmental movements during postural perturbations in humans. Exp Brain Res 72 (3):593-604 